OUTP-03-34P

HEP-TH/0312167

DECEMBER 2003

\title{
ANTHROPICS VERSUS DETERMINISM IN QUANTUM GRAVITY
}

\author{
Dedicated to the memory of Ian Kogan \\ ALON E. FARAGGI* \\ Theoretical Physics, University of Oxford, Oxford, OX1 3NP, England
}

\begin{abstract}
Recently the multitude of vacua in string theory have led some authors to advocate the anthropic principle as a possible resolution for the contrived set of parameters that seem to govern our universe. I suggest that string theories should be viewed as effective theories, and hence of limited utility rather than as "theories of everything". I propose that quantum gravity should admit a form of determinism and that the self-dual points under phase-space duality should play a prominent role in the vacuum selection principle.
\end{abstract}

\section{Introduction}

String theory is in a precarious state of affairs. On the one hand the theory clearly shows great promise in providing a consistent theory of perturbative quantum gravity, while at the same time accommodating the gauge and matter structures that are observed experimentally. On the other hand the multitude of string vacua hinders the prospects of extracting unique predictions from the theory, and has led some authors to advocate the anthropic principle as a resolution for understanding the contrived set of parameters that seem to govern our world ${ }^{1}$.

Part of the difficulty stem from the desire to regard string theory as a "theory of everything". However, even if string theory does lead to the final theory, a more cautious approach towards its utilization seems prudent. In

\footnotetext{
*to appear in the proceedings of the second international conference on string phenomenology, 29/7-4/8 2003, durham uk
} 
this respect, while the theory may offer a glimpse into some of the building blocks of quantum gravity and its relation to observable phenomena, the eventual formalism of quantum gravity and its physical picture may be entirely different from our contemporary perception of string theory. The important question is whether string theory reveals some of the basic properties of the yet unknown quantum gravity theory. Our task is therefore to try to extract from string theory what those properties may be.

In this regard we may view string theories as providing consistent approaches to the synthesis of quantum mechanics and gravity. What lesson should we then extract from the existence of a multitude of string vacua. One possible conclusion is that this is a mere reflection of pushing the probability interpretation of quantum mechanics to its logical conclusion. Namely, the different string theories are the inevitable consequence of applying the probability interpretation of quantum mechanics to the spacetime arena. This is of course not new, but a mere example, albeit perhaps in a more advanced framework, of the multiverse interpretation of quantum gravity. Pursuing this interpretation it therefore seems to me that the anthropic principle is the unavoidable result of pushing the probability interpretation of quantum mechanics to its logical conclusion.

What may therefore be at hand is the inevitable clash between the basic physical hypothesis that underly quantum mechanics and classical general relativity. String theory adopts the probability interpretation of quantum mechanics from the outset. String theory may of course be very useful in allowing a perturbative scheme to quantum gravity, and hence enabling detailed approximate calculations to be performed. However, we should not overlook the possibility that the probability interpretation of quantum mechanics is a derived property rather than a fundamental property of quantum gravity.

In this article I would like to suggest that there exist an alternative possibility. I argue that the evidence suggests that quantum gravity should admit a form of determinism. In this context it is proposed that the selfdual points under phase-space duality should play an important role in the vacuum selection mechanism and in the eventual formulation of quantum gravity. This evidence arises from two considerations. The first is the general expectation that the self-dual points under $\mathrm{T}$-duality in toroidal compactifications extremizes the potential for the moduli of the compactified dimensions in superstring theories. It is then extremely intriguing that the most realistic string models constructed to date, the so-called free fermionic models, are in fact built in the vicinity of the self-dual point. The second piece of evidence arises from the relation of self-dual points under 
phase-space duality to the vacuum state, in a modified Hamilton-Jacobi approach to quantum mechanics. These two completely disparate considerations, in my view, provide two crucial hints in the pursuit of quantum gravity. That the fundamental duality is phase-space duality, and that the self-dual points under this duality play a pivotal role in the vacuum selection.

\section{T-duality}

String theory exhibits various forms of dualities, i.e. relation between different theories at large and small radii of the compactified manifold and at strong and weak coupling. The first type is the T-duality ${ }^{2}$. Consider a point particle moving on a compactified dimension $X$, which obeys the condition $X \sim X+2 \pi R m$. Single valuedness of the wave function of the point particle $\Psi \sim \operatorname{Exp}(i P X)$ implies that the momenta in the compact direction is quantized $P=\frac{m}{R}$ with $m \in Z$. Now consider a string moving in the compactified direction. In this case the string can wrap around the compactified dimension and produce stable winding modes. Hence the left and right-moving momenta in the case of the closed string are given by

$$
P_{L, R}=\frac{m}{R} \pm \frac{n R}{\alpha^{\prime}}
$$

and the mass of the string states is given by

$$
\operatorname{mass}^{2}=\left(\frac{n}{R}\right)^{2}+\left(\frac{m R}{\alpha^{\prime}}\right)^{2}
$$

this is invariant under exchange of large and small radius together with the exchange of winding and momentum modes, i.e.

$$
\frac{1}{R} \leftrightarrow \frac{R}{\alpha^{\prime}} \quad \text { with } \quad m \leftrightarrow n
$$

and is an exact symmetry in string perturbation theory. Furthermore, there exist the self-dual point,

$$
R=\frac{\alpha^{\prime}}{R}
$$

which is the symmetry point under T-duality. In the case of the supersymmetric string on a compactified coordinate the $\mathrm{T}$-duality operation interchanges

$$
\begin{aligned}
\text { type IIA } & \Leftrightarrow \text { type IIB } \\
\text { Heterotic } S O(32) & \Leftrightarrow \text { Heterotic } E_{8} \times E_{8}
\end{aligned}
$$


Now, all this is of course well known since the late 80's. However, the following point is not well appreciated. It is also well known that for specific values of its radius, the compactified coordinate can be realized as specific rational conformal field theories propagating on the string world-sheet. In particular, there exist such a value for which a compactified coordinate can be represented in terms of two free Majorana-Weyl fermions. It so happens that, in some normalization, the self-dual point is at $R=1 / \sqrt{2}$ whereas the free fermionic point is at $R=1$. Hence, the two points do not overlap and the free fermionic point does not coincide with the selfdual point ${ }^{3}$. However, this is merely an artifact of the fact that we have been talking here about bosonic string. In the case of the supersymmetric string the two points, in fact, do coincide. This is a remarkable observation for the following reason. While we do not yet know at what value the compactified coordinate are fixed, naively we would expect that they are stabilized around a symmetry point or at infinity. The self-dual point under T-duality is precisely such a symmetry point. Hence, near the self-dual point, which is the symmetry point under $\mathrm{T}$-duality and around which we may expect that the compactified dimensions stabilize, we can represent the compact dimension as a pair of free Majorana-Weyl fermions propagating on the string world-sheet. Of course, the real picture may be much more complicated. But as a first approximation this is the naive expectation, based on the symmetry properties of string theory.

\section{Realistic string models}

The class of three generation free fermionic models is therefore constructed precisely in the vicinity of the self-dual point under $\mathrm{T}$-duality! I find this to be an extremely intriguing coincidence! I elaborate briefly here on the properties of these models and why they may be considered the most realistic models constructed to date.

From the Standard Model data we may hypothesis that the string vacuum should possess two key properties. The existence of three generations and their embedding in $S O(10)$ representations. The only perturbative string theory that preserves the $S O(10)$ embedding is the heterotic string, because this is the only one that produces the chiral 16 representation of $S O(10)$ in the perturbative spectrum. A class of heterotic string models that accommodate these two key ingredients of the Standard Model spectrum are the so called free fermionic models. As already discussed above the free fermionic point in the moduli space of superstring theories coincides with the self-dual point. The other key property of the free fermionic models is their relation to $Z_{2} \times Z_{2}$ orbifold compactification. While the 
space of possible string compactifications may be beyond count, it seems to me that any model, or class of models, that exhibit realistic properties deserve to be studied in depth.

The structure of the $Z_{2} \times Z_{2}$ orbifold naturally correlates the existence of three generation with the underlying geometry. This arises due to the fact that the $Z_{2} \times Z_{2}$ orbifold has exactly three twisted sectors. Each of the light chiral generations then arises from a distinct twisted sector. Hence, in these models the existence of three generations in nature is seen to arise due to the fact that we are dividing a six dimensional compactified manifold into factors of 2 . In simplified terms, three generations is an artifact of $\frac{6}{2}=1+1+1$. One may further ask whether there is a reason that the $Z_{2}$ orbifold would be preferred versus higher orbifolds. Previously we argued that the free fermionic point coincides with the selfdual point under $T$-duality, which is where we would naively expect the compactified dimensions to stabilize. The special property of the $Z_{2}$ orbifold that sets it apart from higher orbifolds, is the fact that the $Z_{2}$ orbifold is the only one that acts on the coordinates as real coordinates rather than complex coordinates. Furthermore, a classification of the $Z_{2} \times Z_{2}$ orbifold with symmetric shifts on the internal tori reveals that the three generation models in this class are not obtained solely with symmetric shifts on complex tori ${ }^{4}$. The three generation models necessarily employ an asymmetric shift on the internal coordinates. The necessity to incorporate an asymmetric shift in the reduction to three generations, has profound implications for the issues of moduli stabilization and vacuum selection. The reason being that it can only be implemented at enhanced symmetry points in the moduli space. In this context we envision again that the self-dual point under $\mathrm{T}$-duality plays a special role. In the context of nonperturbative dualities the dilaton and moduli are interchanged, with potentially important implications for the problem of dilaton stabilization.

To summarize this section. The argument here is that T-duality is the pivotal property of string theory in trying to understand the vacuum selection mechanism. In this context the self-dual points may play an important role. It is then extremely intriguing that precisely in the vicinity of the self-dual point there exist a class of models that capture the two main characteristics of the Standard Model. The existence of three generations together with their $S O(1 O)$ embedding.

\section{Phase-space duality}

Duality and self-duality also play a key role in the recent formulation of quantum mechanics from an equivalence postulate ${ }^{7}$. An important facet 
of this formalism is the phase--space duality, which is manifested due to the involutive nature of the Legendre transformation. In the Hamilton-Jacobi formalism of classical mechanics the phase-space variables are related by Hamilton's generating function $p=\partial_{q} S_{0}(q)$. One then obtains the dual Legendre transformations ${ }^{7}$,

$$
S_{0}=p \partial_{p} T_{0}-T_{0}
$$

and

$$
T_{0}=q \partial_{q} S_{0}-S_{0},
$$

where $T_{0}(p)$ is a new generating function defined by $q=\partial_{p} T_{0}$. Two points are important to note. The first is that because the Legendre transformation is not defined for linear functions, i.e. for physical systems with $S_{0}=A q+B$, implies that the Legendre duality fails for the free system and for the free system with vanishing energy. The second is that we can associate a second order differential equation with each Legendre transformation ${ }^{7}$. There exist then a set of solutions, labeled by $p q=\gamma$, where $\gamma$ is a constant to be determined, which are simultaneous solutions of the two sets of differential equations. These are the self dual states under the phase-space duality.

\section{The quantum equivalence postulate}

The Legendre phase-space duality and its breakdown for the free system are intimately related to the equivalence postulate, which states that all physical systems labeled by the function $W(q)=V(q)-E$, can be connected by a coordinate transformation, $q^{a} \rightarrow q^{b}=q^{b}\left(q^{a}\right)$, defined by $S_{0}^{b}\left(q^{b}\right)=S_{0}^{a}\left(q^{a}\right)$. This postulate implies that there always exist a coordinate transformation connecting any state to the state $W^{0}\left(q^{0}\right)=0$. Inversely, this means that any physical state can be reached from the state $W^{0}\left(q^{0}\right)$ by a coordinate transformation. This postulate cannot be consistent with classical mechanics. The reason being that in Classical Mechanics (CM) the state $W^{0}\left(q^{0}\right) \equiv 0$ remains a fixed point under coordinate transformations. Thus, in $\mathrm{CM}$ it is not possible to generate all states by a coordinate transformation from the trivial state. Consistency of the equivalence postulate implies the modification of CM, which is analyzed by a adding a still unknown function $Q$ to the Classical Hamilton-Jacobi Equation (CHJE). Consistency of the equivalence postulate fixes the transformation properties for $W(q)$,

$$
W^{v}\left(q^{v}\right)=\left(\partial_{q^{v}} q^{a}\right)^{2} W^{a}\left(q^{a}\right)+\left(q^{a} ; q^{v}\right),
$$


and for $Q(q)$,

$$
Q^{v}\left(q^{v}\right)=\left(\partial_{q^{v}} q^{a}\right)^{2} Q^{a}\left(q^{a}\right)-\left(q^{a} ; q^{v}\right),
$$

which fixes the cocycle condition for the inhomogeneous term

$$
\left(q^{a} ; q^{c}\right)=\left(\partial_{q^{c}} q^{b}\right)^{2}\left[\left(q^{a} ; q^{b}\right)-\left(q^{c} ; q^{b}\right)\right] .
$$

The cocycle condition is invariant under Möbius transformations and fixes the functional form of the inhomogeneous term. The cocycle condition is generalizable to higher, Euclidean or Minkowski, dimensions, where the Jacobian of the coordinate transformation extends to the ratio of momenta in the transformed and original systems ${ }^{7}$.

The identity

$$
\left(\partial_{q} S_{0}\right)^{2}=\hbar^{2} / 2\left(\left\{\exp \left(i 2 S_{0} / \hbar, q\right)\right\}-\left\{S_{0}, q\right\}\right),
$$

which embodies the equivalence postulate, leads to the Schrödinger equation. Making the identification

$$
W(q)=V(q)-E=-\hbar^{2} / 4 m\left\{\mathrm{e}^{\left(i 2 S_{0} / \hbar\right)}, q\right\},
$$

and

$$
Q(q)=\hbar^{2} / 4 m\left\{S_{0}, q\right\},
$$

we have that $S_{0}$ is solution of the Quantum Stationary Hamilton-Jacobi Equation (QSHJE),

$$
(1 / 2 m)\left(\partial_{q} S_{0}\right)^{2}+V(q)-E+\left(\hbar^{2} / 4 m\right)\left\{S_{0}, q\right\}=0,
$$

where $\{$,$\} denotes the Schwarzian derivative. From the identity we deduce$ that the trivializing map is given by $q \rightarrow \tilde{q}=\psi^{D} / \psi$, where $\psi^{D}$ and $\psi$ are the two linearly independent solutions of the corresponding Schrödinger equation ${ }^{7}$. We see that the consistency of the equivalence postulate forces the appearance of quantum mechanics and of $\hbar$ as a covariantizing parameter.

\section{The role of the self-dual states}

The remarkable property of the QSHJE, which distinguishes it from the classical case, is that it admits non-trivial solution also for the trivial state, $W(q) \equiv 0$. In fact the QSHJE implies that $S_{0}=$ constant is not an allowed solution. The fundamental characteristic of quantum mechanics in this approach is that $S_{0} \neq A q+B$. Rather, the solution for the ground state, with $V(q)=0$ and $E=0$, is given by

$$
S_{0}=i \hbar / 2 \ln q,
$$


up to Möbius transformations. Remarkably, this quantum ground state solution coincides with the self-dual state of the Legendre phase-space transformation and its dual. Thus, we have that the quantum self-dual state plays a pivotal role in ensuring both the consistency of the equivalence postulate and definability of the Legendre phase-space duality for all physical states. The association of the self-dual state and the physical state with $V(q)=0$ and $E=0$ provides a hint that the equivalence postulate and Legendre phase-space duality may shed new light on the nature of the vacuum.

Two additional properties of the formalism are important to note. The first is the existence of an intrinsic length scale, which is strictly related to the phase-space duality. Considering solutions of the basic Schrödinger equation with $V(q)=E=0$, i.e. $\partial_{q}^{2} \psi^{0}=0$, one has to introduce a length to consider linear combinations of $\psi^{D^{0}}=q^{0}$ and $\psi^{0}=1$. Since in this case $W \equiv 0$, there is no scale in the Schrödinger problem, and a universal length scale is enforced. The second is the existence of equivalence classes of the wave-function. As the QHJE is a third-order differential equation whereas the Schrödinger equation is a second order one, more initial conditions are needed to be specified in the case of the QHJE. It follows that the wave function remains invariant under suitable transformations of $\delta=\{\alpha, \ell\}$, corresponding to different deterministic trajectories. The implication is that there are hidden variables which depend of the Planck length and that these can suitably change without affecting the wave-function. The probabilistic nature of the wave-function may therefore arise due to our ignorance of the underlying Planck scale physics.

\section{Conclusions}

It is proposed that phase-space duality is the guiding property in trying to formulate quantum gravity. In this respect $\mathrm{T}$-duality is a key property of string theory. We can think of T-duality as a phase-space duality in the sense that we are exchanging momenta and winding modes in compact space. We can turn the table around and say that the key feature of string theory is that it preserves the phase-space duality in the compact space. Namely, prior to compactification the wave-function of a point particle $\Psi \sim \operatorname{Exp}(i P X)$ is invariant under $p \leftrightarrow x$. However, in the ordinary KaluzaKlein compactification this invariance is lost due to the quantization of the momentum modes. String theory restores this invariance by introducing the winding modes. It is further argued that the self-dual points under phase-space duality are intimately connected to the choice of the vacuum. 
The evidence for this arises from the phenomenological success of the free fermionic models that are constructed in the vicinity of the self-dual point, as well as from the formal derivation of quantum mechanics from phasespace duality and the equivalence postulate.

\section{Acknowledgments}

This work was supported in part by the PPARC and by the Royal Society.

\section{References}

1. L. Susskind, hep-th/0302219; M.R. Douglas, JHEP 0305, 046 (2003); T. Banks, M. Dine, E. Gorbatov, hep-th/0309170; J.F. Donoghue, hepth/0310203.

2. For review and references see e.g.: A. Giveon et al: Phys. Rep. 244, 77 (1994).

3. P. Ginsparg: Nucl. Phys. B 295, 153 (1988)

4. A.E. Faraggi, C. Kounnas, S.E.M. Nooij and J. Rizos, hep-th/0311058.

5. I. Antoniadis, C.P. Bachas and C. Kounnas, Nucl. Phys. B 289, 87 (1987); H. Kawai, D.C. Lewellen and S.H. Tye, Nucl. Phys. B 288, 1 (1987).

6. I. Antoniadis, J.R.Ellis, J.S. Hagelin and D.V. Nanopoulos, Phys. Lett. B 231, 65 (1989); A.E. Faraggi, D.V. Nanopoulos and K.J. Yuan, Nucl. Phys. B 335, 347 (1990); I. Antoniadis, G.K. Leontaris and J. Rizos, Phys. Lett. B 245, 161 (1990); A.E. Faraggi, Phys. Lett. B 278, 131 (1992); Nucl. Phys. B 387, 239 (1992); G.B. Cleaver, A.E. Faraggi and D.V. Nanopoulos, Phys. Lett. B 455, 135 (1999).

7. A.E. Faraggi and M. Matone, Phys. Rev. Lett. 78, 163 (1997); Phys. Lett. B 450, 34 (1999); Phys. Lett. B 437, 369 (1998); Phys. Lett. A 249, 180 (1998); Phys. Lett. B 445, 77 (1998); Phys. Lett. B 445, 357 (1999); Int. J. Mod. Phys. A 15, 1869 (2000); G. Bertoldi, A.E. Faraggi and M. Matone, Class. Quant. Grav. 173965 (2000).

8. E.R. Floyd, Phys. Rev. D 25, 1547 (1982); Phys. Rev. D 26, 1339 (1982); Phys. Rev. D 29, 1842 (1984); Phys. Rev. D 34, 3246 (1986); Phys. Lett. A 214, 259 (1996); Int. J. Mod. Phys. A 14, 1111 (1999). 